\title{
Avaliação do desempenho de estudantes de medicina no OSCE: o papel do estudo prévio
}

\author{
Performance evaluation of medical students in the OSCE: the role of previous study \\ Evaluación del desempeño de estudiantes de medicina en la OSCE: el papel del estudio \\ previo
}

Amanda Santos Duarte ${ }^{1}$, Adenilso Pinheiro da Silva ${ }^{1}$, Artur dos Santos Soares ${ }^{1 *}$, Ana Bárbara Barbosa Roque ${ }^{1}$, Joel Ferreira de Jesus Neto ${ }^{1}$, Celina Cláudia Israel Sefer ${ }^{1}$.

\section{RESUMO}

Objetivo: Avaliar como os estudantes de Medicina do ciclo básico se preparam para realizar o OSCE. Métodos: Estudo transversal, analítico, quantitativo e qualitativo no qual foram aplicados questionários com perguntas diretas e respostas objetivas aos acadêmicos de Medicina do $1^{\circ}$ ao $4^{0}$ semestre do curso de Medicina. Sendo a amostragem probabilística 166 alunos cursando o ciclo básico no período de setembro a dezembro de 2019, na qual foi usado uns questionários próprios autoaplicáveis, onde serão abordadas perguntas pertinentes referentes aos métodos de estudo, ao tempo destinado aos estudos, grau de ansiedade e de confiança para a realização do OSCE. Resultados: O sexo feminino foi o de maior incidência significante $\left({ }^{*} p<0.0001\right.$ ) entre os discentes entrevistados $(69.3 \%)$. Houve diferença significante $\left({ }^{*} p<0.001\right)$ entre os períodos estudados, em relação a faixa etária atual dos discentes pesquisados. Conclusão: Comparando-se os 4 semestres do ciclo básico: os métodos de estudos utilizados em maior proporção foram as anotações e os livros; a quantidade de tempo destinada aos estudos variou proporcionalmente entre 2 a 4 horas.

Palavras-chave: Habilidades para realização de testes, Epidemiologia, Medicina.

\section{ABSTRACT}

Objective: To evaluate how medical students in the basic cycle prepare to perform the OSCE. Methods: Crosssectional, analytical, quantitative and qualitative study in which questionnaires with direct questions and objective answers were applied to medical students from the 1 st to the 4 th semester of the medical course. As the probabilistic sampling 166 students attending the basic cycle in the period from September to December 2019, in which a selfadministered questionnaire was used, where pertinent questions regarding the study methods, the time allocated to studies, degree of anxiety and confidence for the OSCE. Results: The female gender was the one with the highest significant incidence $\left({ }^{*} p<0.0001\right)$ among the interviewed students $(69.3 \%)$. There was a significant difference $\left({ }^{*} p\right.$ $<0.001$ ) between the periods studied, in relation to the current age range of the students surveyed. Conclusion: Comparing the 4 semesters of the basic cycle: the study methods used in the greatest proportion were notes and books; the amount of time allocated to the studies varied proportionally between 2 and 4 hours.

Key words: Test talking skills, Epidemiology, Medicine.

\section{RESUMEN}

Objetivo: Evaluar cómo los estudiantes de medicina en el ciclo básico se preparan para realizar la OSCE. Métodos: Estudio transversal, analítico, cuantitativo y cualitativo en el que se aplicaron cuestionarios con preguntas directas y respuestas objetivas a estudiantes de medicina del $1^{\circ}$ al $4^{\circ}$ semestre del curso de medicina. Como muestra probabilística, 166 estudiantes asistieron al ciclo básico en el período de septiembre a diciembre de 2019, en el que se utilizó un cuestionario autoadministrado, donde se formularon preguntas pertinentes sobre los métodos de estudio, el tiempo asignado a los estudios, el grado de ansiedad y confianza para la OSCE. Resultados: El sexo femenino fue el que tuvo la mayor incidencia significativa ( ${ }^{*} p<0.0001$ ) entre los estudiantes entrevistados $(69.3 \%)$. Hubo una diferencia significativa ${ }^{*} p<0.001$ ) entre los períodos estudiados, en relación con el rango de edad actual de los estudiantes encuestados. Conclusión: Comparando los 4 semestres del ciclo básico: los métodos de estudio utilizados en la mayor proporción fueron notas y libros; El tiempo asignado a los estudios varió proporcionalmente entre 2 y 4 horas.

Palabras clave: Habilidades para tomar exámenes, Epidemiología, Medicina.

${ }^{1}$ Centro Universitário Metropolitano da Amazônia (UNIFAMAZ), Belém - PA.

*E-mail: artur_soares_@hotmail.com

SUBMETIDO EM: $7 / 2020$

ACEITO EM: 8/2020

PUBLICADO EM: 10/2020 


\section{INTRODUÇÃO}

O Exame Clínico Objetivo e Estruturado (OSCE) foi criado e implementado pela Universidade de Dundee, na Escócia, em 1975, mas somente foi implantado no Brasil em 2014 através da Resolução CNE ㄲo3/2014 do Ministério da Educação que institui diretrizes curriculares nacionais do curso de graduação de Medicina, na qual é ressaltada a aprendizagem crítica-reflexiva, em que os acadêmicos devem participar ativamente do processo de ensino e aprendizagem por meio de habilidades, conhecimento e atitude crítica (NEVES RS, et al., 2017; ZIMMERMANN MH, 2019).

O uso do OSCE na educação médica tornou-se o método mais utilizado para a avaliação da competência clínica. Este método visa avaliar habilidades clínicas (anamnese, exame físico, habilidades para raciocínio clínico, diagnóstico e tratamento), conhecimento, atitudes, profissionalismo e comunicação (JOONG HS, et al., 2015; FRANCOS CAGS, et al., 2015).

Durante o processo de preparo para a realização do OSCE o acadêmico utiliza de diversos meios para se sentir confiante diante desta avaliação, como é um método extremamente prático, o acadêmico necessita reunir o seu conhecimento prévio com estudos científicos e aplicá-los em uma forma prática. Dessa forma, o autoestudo é necessário (RADHIKA G, et al., 2015; COMERT M, et al., 2017; GRAF J, et al., 2015).

$\mathrm{O}$ autoestudo é quando o acadêmico utiliza meios para estudar sozinho. Isso possibilita que o aluno preencha lacunas do seu conhecimento, que não necessariamente é uma dúvida gerada em todos os alunos. Dessa maneira, ele focaliza nas áreas que estão deficitárias e melhora outras através do seu estudo direcionado por si mesmo. Isto é fundamental para os médicos atualmente, pois o torna um aluno aprendiz ao longo da vida (BESTETTI RB, et al., 2017).

O OSCE é uma forma de avaliar as habilidades e competências dos alunos durante sua formação médica, por isso este trabalho tem como objetivo analisar a percepção do estudante do Centro Universitário Metropolitano da Amazônia (UNIFAMAZ) sobre essa prática, bem como utilizar processos meta-avaliativos para a qualificação desses exames. O objetivo do presente artigo foi avaliar como os estudantes de Medicina do ciclo básico se preparam para realizar o OSCE.

\section{MÉTODOS}

Este estudo foi livremente baseado no trabalho do Brian Mavis (Does Studying for na objective strutured clinical examination make a difference?). Estudo transversal, analítico, quantitativo e qualitativo no qual foram aplicados questionários com perguntas diretas e respostas objetivas aos acadêmicos de Medicina do $1^{\circ}$ ao $4^{\circ}$ semestre do curso de Medicina do Centro Universitário Metropolitano da Amazônia (UNIFAMAZ), no período de setembro a dezembro de 2019.

O estudo foi realizado nos ambulatórios do Eixo Habilidades Clínicas do $1^{\circ}$ ao $4^{\circ}$ semestre da UNIFAMAZ. A pesquisa foi realizada com os acadêmicos do $1^{\circ}$ ao $4^{\circ}$ semestre do curso de Medicina da UNIFAMAZ, estando esses regularmente matriculados no curso.

Foram incluídos na pesquisa todos os alunos que concordaram em participar da mesma, após esclarecimentos dos seus objetivos, métodos, riscos e benefícios, e que estavam regularmente matriculados no curso do 1 ao 4 semestre.

Foram excluídos desta pesquisa os acadêmicos que responderam o questionário de forma incompleta, que desistiram do curso de Medicina ao longo da pesquisa e os que não completaram todas as etapas das avaliações necessárias. Não foram incluídas no estudo as turmas a partir do 5ํㅗ semestre, em virtude de ser uma avaliação somente do ciclo básico.

Sendo a amostragem probabilística 166 alunos cursando o ciclo básico no período de setembro a dezembro de 2019, na qual foi usado um questionário autoaplicável, onde serão abordadas perguntas pertinentes referentes aos métodos de estudo, ao tempo destinado aos estudos, grau de ansiedade e de confiança para a realização do OSCE. 
O questionário tem perguntas objetivas sobre gênero, idade (15-19, 20-24, 25-29, >30), período do curso, método utilizado para o estudo prévio (livros, anotações, artigos, prática, audiovisual, testes e mapa conceitual, o tempo por dia destinado ao estudo ( $<1$ hora, 2 horas, 3 horas, $>4$ horas), o grau de ansiedade antes do OSCE (nada, pouco, moderado e extremamente).

Após o questionário ser aplicado, foi feito uma segunda análise sobre as notas dos acadêmicos avaliados antes do OSCE, para avaliar o desempenho foi definido como "Insuficiente" a nota < 4, "Regular" a nota 5-6, "Bom" a nota 7-8 e "Excelente" a nota 9-10.Foram aplicados os questionários (ANEXO A) para os 166 acadêmicos da UNIFAMAZ, do $1^{\circ}$ ao $4^{\circ}$ semestre, juntamente com o TCLE, durante o confinamento préOSCE.

Os resultados dos dados foram catalogados em bancos para criação de tabelas e gráficos. As variáveis quantitativas foram expressas por média e desvio padrão e as qualitativas foram ajustadas através de frequência e percentual. O programa estatístico Bioestat 5.0 foi utilizado para a análise dos dados, adotando $\alpha=0,05$ ou $5 \%$ com um intervalo de confiança de $95 \%$. Usaram-se os testes não paramétricos qui-quadrado.

Essa pesquisa foi submetida e aceito pelo Comitê de Ética em Pesquisa do Centro Universitário Metropolitano da Amazônia (UNIFAMAZ) para fins de análise e autorização da pesquisa. O número do CAAE é 13583819.3.0000.5701. Os pacientes envolvidos foram estudados segundo os preceitos da Declaração de Helsinki e do Código de Nuremberg, respeitada a resolução 466/12 do Conselho Nacional de Saúde (CNS).

\section{RESULTADOS}

O sexo feminino foi o de maior incidência significante $\left({ }^{*} p<0.0001\right)$ entre os discentes entrevistados (69.3\%). Na comparação entre os períodos analisados, não houve diferença significante $(p=0.2829)$ na proporção entre os gêneros de acordo com os períodos do curso tendo o $n=115$.

Tabela 1 - Discentes segundo o gênero, de acordo com o período do curso, UNIFAMAZ, 2019.

\begin{tabular}{ccccc}
\hline \multirow{2}{*}{ Período do curso } & \multicolumn{2}{c}{ Feminino* $^{*}$} & \multicolumn{2}{c}{ Masculino } \\
\cline { 2 - 5 } & Freq & $\%$ & Freq & $\%$ \\
\hline Primeiro & 19 & $16.5 \%$ & 14 & $27.5 \%$ \\
Segundo & 32 & $27.8 \%$ & 16 & $31.4 \%$ \\
Terceiro & 31 & $27.0 \%$ & 11 & $21.6 \%$ \\
Quarto & 33 & $28.7 \%$ & 10 & $19.6 \%$ \\
\hline Total & $\mathbf{1 1 5}$ & $\mathbf{6 9 . 3} \%$ & $\mathbf{5 1}$ & $\mathbf{3 0 . 7} \%$ \\
\hline
\end{tabular}

Legenda: $p=0.2829$ Teste Qui-Quadrado Independência* $p<0.0001$ Teste Qui-Quadrado Aderência. Fonte: Soares AS, et al., 2020.

Houve diferença significante ( ${ }^{*} p<0.001$ ) entre os períodos estudados, em relação a faixa etária atual dos discentes pesquisados. Os discentes do primeiro período, como já era esperado, se encontram em maior proporção (54.5\%) na faixa entre 15 a 19 anos e a maior proporção, entre todas as faixas, foi observada entre 20 a 24 anos nos alunos do quarto ano (81.4\%).

Em relação ao método utilizado pelos discentes para estudo, foi encontrada diferença significante $\left({ }^{*} \mathrm{p}=\right.$ 0.0095) nas proporções entre os períodos pesquisados. Os métodos com maior proporção entre os alunos foram as anotações e os livros para o primeiro (72.7\% e $84.8 \%)$, segundo (79.2\% para ambos) e terceiro (85.7\% e $76.2 \%$ ) períodos respectivamente; já o quarto período opinou em primeiro lugar pela prática (69.8\%), seguido pelas anotações (62.8\%). 
Tabela 2 - Discentes segundo o método utilizado para estudo, de acordo com o período do curso, UNIFAMAZ, 2019.

\begin{tabular}{ccccccccc}
\hline \multirow{2}{*}{ Método de estudo } & \multicolumn{8}{c}{ Período do curso } \\
\cline { 2 - 9 } & \multicolumn{2}{c}{ Primeiro } & \multicolumn{2}{c}{ Segundo } & \multicolumn{2}{c}{ Terceiro } & Quarto \\
\hline Anotações & 24 & $72.7 \%$ & 38 & $79.2 \%$ & 36 & $85.7 \%$ & 27 & $62.8 \%$ \\
Livros & 28 & $84.8 \%$ & 38 & $79.2 \%$ & 32 & $76.2 \%$ & 15 & $34.9 \%$ \\
Prática & 16 & $48.5 \%$ & 28 & $58.3 \%$ & 26 & $61.9 \%$ & 30 & $69.8 \%$ \\
Audiovisual & 17 & $51.5 \%$ & 27 & $56.3 \%$ & 22 & $52.4 \%$ & 8 & $18.6 \%$ \\
Artigos & 12 & $36.4 \%$ & 13 & $27.1 \%$ & 18 & $42.9 \%$ & 7 & $16.3 \%$ \\
Mapa conceitual & 13 & $39.4 \%$ & 13 & $27.1 \%$ & 13 & $31.0 \%$ & 11 & $25.6 \%$ \\
Testes & 8 & $24.2 \%$ & 4 & $8.3 \%$ & 7 & $16.7 \%$ & 10 & $23.3 \%$ \\
\hline
\end{tabular}

Legenda: * $p=0.0095$ Teste Qui-Quadrado Partição. Fonte: Soares AS, et al., 2020.

O tempo por dia destinado aos estudos de habilidades clínicas apresentou proporções significativamente diferentes $\left({ }^{*} p=0.0045\right)$, mostrando um maior tempo de estudo pelos discentes do quarto período que destinam de 03 a mais de 04 horas (69.0\%) seguido os alunos do terceiro período que utilizaram de 03 a mais de 04 horas ao estudo (66,7\%). Já os alunos do primeiro e segundo período, utilizam de 02 a 03 horas de estudo para este assunto (78.8\% e $64,6 \%$, respectivamente).

Tabela 3 - Discentes segundo o tempo utilizado para estudos de habilidades clínicas. de acordo com o período do curso, UNIFAMAZ, 2019.

\begin{tabular}{ccccccccc}
\hline \multirow{2}{*}{$\begin{array}{c}\text { Tempo que utiliza } \\
\text { para estudos }\end{array}$} & \multicolumn{7}{c}{ Primeiro } & \multicolumn{2}{c}{ Segundo } & \multicolumn{2}{c}{ Terceiro } & Quarto \\
\cline { 2 - 10 } & 4 & $12.1 \%$ & 4 & $8.3 \%$ & 2 & $4.8 \%$ & 0 & $0.0 \%$ \\
$<=01$ & 17 & $51.5 \%$ & 11 & $22.9 \%$ & 12 & $28.6 \%$ & 9 & $20.9 \%$ \\
02 & 9 & $27.3 \%$ & 20 & $41.7 \%$ & 13 & $31.0 \%$ & 25 & $58.1 \%$ \\
03 & 3 & $9.1 \%$ & 13 & $27.1 \%$ & 15 & $35.7 \%$ & 9 & $20.9 \%$ \\
$>=04$ & $\mathbf{3 3}$ & $\mathbf{1 9 . 9} \%$ & $\mathbf{4 8}$ & $\mathbf{2 8 . 9} \%$ & $\mathbf{4 2}$ & $\mathbf{2 5 . 3} \%$ & $\mathbf{4 3}$ & $\mathbf{2 5 . 9 \%}$ \\
\hline Total & &
\end{tabular}

Legenda: ${ }^{*} p=0.0045$ Teste Qui-Quadrado Partição. Fonte: Soares AS, et al., 2020.

A ansiedade que os discentes sentem antes da realização do OSCE, não apresentou variação proporcional significativa $(p=0.6474)$ entre os períodos estudados. A maioria significante $\left({ }^{*} p<0.001\right)$ se declaram "Extremamente" ansiosos com percentuais entre $57.1 \%$ no primeiro período e $67.4 \%$ no quarto.

Tabela 4 - Discentes segundo o nível de ansiedade antes do OSCE, de acordo com o período do curso, UNIFAMAZ, 2019.

\begin{tabular}{ccccccccc}
\hline \multirow{2}{*}{$\begin{array}{c}\text { Período do } \\
\text { curso }\end{array}$} & \multicolumn{6}{c}{ Nada } & \multicolumn{2}{c}{ O quão ansioso você se sente antes do OSCE: } \\
\cline { 2 - 9 } & \multicolumn{1}{c}{ Nouco } & \multicolumn{2}{c}{ Muito } & \multicolumn{2}{c}{ Extremamente $^{*}$} \\
\hline Primeiro & 0 & $0.0 \%$ & 4 & $12.1 \%$ & 9 & $27.3 \%$ & 20 & $60.6 \%$ \\
Segundo & 2 & $4.2 \%$ & 2 & $4.2 \%$ & 12 & $25.0 \%$ & 32 & $66.7 \%$ \\
Terceiro & 2 & $4.8 \%$ & 7 & $16.7 \%$ & 9 & $21.4 \%$ & 24 & $57.1 \%$ \\
Quarto & 1 & $2.3 \%$ & 6 & $14.0 \%$ & 7 & $16.3 \%$ & 29 & $67.4 \%$ \\
\hline Total & $\mathbf{5}$ & $\mathbf{3 . 0 \%}$ & $\mathbf{1 9}$ & $\mathbf{1 1 . 4 \%}$ & $\mathbf{3 7}$ & $\mathbf{2 2 . 3 \%}$ & $\mathbf{1 0 5}$ & $\mathbf{6 3 . 3 \%}$ \\
\hline
\end{tabular}

Legenda: $p=0.6474$ Teste Qui-Quadrado Partição* $p<0.0001$ Teste Qui-Quadrado Aderência.

Fonte: Soares AS, et al., 2020.

Quanto a confiança que os discentes sentem em relação aos conteúdos estudados, não houve diferença proporcional significante $(p=0.0985)$ entre os períodos. As maiores proporções se concentraram entre "Pouco" e "Muito" (48.8\% e 42.2\%) respectivamente. O quarto período mostrou maior proporção $(51.2 \%)$ em "Muito" e o segundo período em "Pouco" (64.6\%). 
Tabela 5 - Discentes segundo ao grau de confiança em relação aos assuntos estudados, de acordo com o período do curso, UNIFAMAZ, 2019.

\begin{tabular}{ccccccccc}
\hline $\begin{array}{c}\text { Período do } \\
\text { curso }\end{array}$ & \multicolumn{3}{c}{ O quão confiante você se sente em relação aos conteúdos estudados: } \\
\cline { 2 - 9 } Nada & \multicolumn{3}{c}{ Pouco } & \multicolumn{2}{c}{ Muito } & \multicolumn{2}{c}{ Extremamente } \\
\hline Primeiro & 1 & $3.0 \%$ & 15 & $45.5 \%$ & 15 & $45.5 \%$ & 2 & $6.1 \%$ \\
Segundo & 2 & $4.2 \%$ & 31 & $64.6 \%$ & 14 & $29.2 \%$ & 1 & $2.1 \%$ \\
Terceiro & 2 & $4.8 \%$ & 17 & $40.5 \%$ & 19 & $45.2 \%$ & 4 & $9.5 \%$ \\
Quarto & 1 & $2.3 \%$ & 18 & $41.9 \%$ & 22 & $51.2 \%$ & 2 & $4.7 \%$ \\
\hline Total & $\mathbf{6}$ & $\mathbf{3 . 6 \%}$ & $\mathbf{8 1}$ & $\mathbf{4 8 . 8} \%$ & $\mathbf{7 0}$ & $\mathbf{4 2 . 2} \%$ & $\mathbf{9}$ & $\mathbf{5 . 4 \%}$ \\
\hline
\end{tabular}

Legenda: $p=0.0985$ Teste Qui-Quadrado Partição* $p<0.0001$ Teste Qui-Quadrado Aderência.

Fonte: Soares AS, et al., 2020.

$\mathrm{Na}$ análise do desempenho no OSCE dos discentes foi encontrado diferença proporcional significante ( $\mathrm{p}$ $=0.0302$ ) entre os períodos pesquisados. Apenas $6.3 \%$ dos alunos do segundo período alcançaram um desempenho "Excelente". A maioria dos discentes, em todos os períodos atingiram um desempenho "Bom", com proporções acima de $50 \%$. No geral, o conceito "Bom" foi o que apresentou percentual significante ( ${ }^{*} p<$ $0.0001)$ em relação aos demais $(59.0 \%)$.

Tabela 6 - Discentes segundo o desempenho alcançado no OSCE, de acordo com o período do curso, UNIFAMAZ, 2019.

\begin{tabular}{|c|c|c|c|c|c|c|c|c|}
\hline \multirow{2}{*}{$\begin{array}{l}\text { Período do } \\
\text { curso }\end{array}$} & \multicolumn{8}{|c|}{ Desempenho / Conceito } \\
\hline & \multicolumn{2}{|c|}{ Insuficiente } & \multicolumn{2}{|c|}{ Regular } & \multicolumn{2}{|c|}{ Bom $^{*}$} & \multicolumn{2}{|c|}{ Excelente } \\
\hline Primeiro & 0 & $0.0 \%$ & 4 & $12.1 \%$ & 18 & $54.6 \%$ & 11 & $33.3 \%$ \\
\hline Segundo & 2 & $4.1 \%$ & 16 & $33.3 \%$ & 27 & $56.3 \%$ & 3 & $6.3 \%$ \\
\hline Terceiro & 0 & $0.0 \%$ & 6 & $14.3 \%$ & 26 & $61.9 \%$ & 10 & $23.8 \%$ \\
\hline Quarto & 2 & $4.7 \%$ & 7 & $16.3 \%$ & 27 & $62.8 \%$ & 7 & $16.3 \%$ \\
\hline Total & 4 & $2.4 \%$ & 33 & $19.9 \%$ & 98 & $59.0 \%$ & 31 & $18.7 \%$ \\
\hline
\end{tabular}

Legenda: ${ }^{*} p=0.0302$ Teste Qui-Quadrado Partição* $p<0.0001$ Teste Qui-Quadrado Aderência.

Fonte: Soares AS, et al., 2020.

Em relação a nota atribuída ao desempenho no OSCE, o valor variou de 1.55 a 10.0, ou seja, a amplitude foi muito alta, com uma diferença estatisticamente significante $\left({ }^{*} p=0.0014\right)$. O quarto período foi o que apresentou maior variação ( $D P \pm 1.56)$. A menor média alcançada foi a do segundo período (7.36) e a maior foi a do primeiro período (8.33).

\section{DISCUSSÃO}

Os resultados apresentados neste estudo ressaltam sua contribuição ao debate sobre a educação médica, traçando o perfil dos discentes do curso de Medicina do Centro Universitário Metropolitano da Amazônia (UNIFAMAZ), a fim de avaliar como acontece o estudo prévio, a influência da ansiedade e da autoconfiança, além no desempenho de estudantes de Medicina do ciclo básico, no OSCE.

A análise dos resultados, nos mostram que $69.3 \%$ dos discentes da amostra em questão, são do sexo feminino; esse resultado vai ao encontro de achados de estudos anteriores, que o apontam como maior prevalência entre alunos de Medicina por fatores não explicados (SCHEFFER M, et al. 2018; PAES AT, et al. 2018; ALVARENGA VC, et al. 2019). No entanto, outros estudos divergem da informação, indicando a prevalência masculina entre o perfil dos estudantes, além de estudos que demostram um equilíbrio entre o percentual de homens e mulheres (MINELLA LS, 2017; GERMANO JCV, 2018; MESSIAS AJ, et al. 2018). Dessa forma, foi possível analisar que a prevalência maior de um determinado sexo estar na graduação depende da região onde a pesquisa foi desenvolvida.

Quanto à idade, os discentes do primeiro período, como já era esperado, encontram-se em maior proporção $(54,5 \%)$ na faixa entre 15 a 19 anos. A maior proporção observada foi entre estudantes do quarto período, na faixa etária de 20 a 24 anos (81,4\%), seguindo a tendência nacional, na qual $73,7 \%$ dos universitários encontra-se nessa faixa etária, apontando o rejuvenescimento no perfil acadêmico das escolas de Medicina no Brasil (FILHO FABC, 2015). Em relação ao método utilizado pelos discentes para estudo nos quatro períodos avaliados, as anotações e os livros obtiveram maior proporção entre os alunos, com valores 
equivalentes a $(72.7 \%$ e $84.8 \%)$ no primeiro período, (79.2\% para ambos) no segundo e $(85.7 \%$ e $76.2 \%)$ no terceiro; já o quarto período opinou em primeiro lugar pela prática $(69.8 \%)$, seguido pelas anotações $(62.8 \%)$.

Estudos anteriores mostram que a escolha deste método está associada à condição dos materiais serem confeccionados pelos próprios alunos, através da transcrição de assuntos abordados pelo professor, tornando as anotações fonte de conteúdo preciso. Além disso, estes estudos também destacam que, apesar da interferência que a relação estudante-livro vem sofrendo, devido ao surgimento de novos suportes informativos, $70,6 \%$ da amostra considerou o livro como a principal fonte de estudo antes da avaliação, indicando que os estudantes o consideram uma fonte de estudo confiável. No entanto, houve uma mudança no padrão de estudo dos discentes no quarto período. Os livros que juntamente com as anotações haviam sido os principais métodos de estudos utilizadas nos três períodos anteriores, foi substituído pela prática.

O tempo por dia destinado aos estudos de habilidades clínicas apresentou proporções significativamente diferentes $\left({ }^{*} p=0.0045\right)$, mostrando um maior tempo de estudo pelos discentes do quarto período que destinam de 03 a mais de 4 horas $(69.0 \%$ ) seguido os alunos do terceiro período que utilizaram de 3 a mais de 04 horas ao estudo $(66,7 \%)$. Já os alunos do primeiro e segundo período, utilizam de 2 a 3 horas de estudo para este assunto $(78.8 \%$ e $64,6 \%$, respectivamente).

Diversos dados na literatura apontam que estudantes com maior tempo dedicado aos estudos/atividades acadêmicas fora do âmbito da faculdade ( $\geq 3$ horas), tendem a não adiar decisões, dificilmente se distraem, não cometem erros nas tarefas atribuídas e apresentam alto escore de engajamento (NETO JAC, 2016; SILVA JOM, 2018; COUTO LB, et al., 2019). Ainda assim, o método utilizado para avaliar o tempo de estudo não proporcionou diferenciar os alunos os quais revisam regularmente os assuntos do curso daqueles que estudam somente mediante a aproximação do OSCE.

No que se refere a ansiedade, a maioria dos estudantes declarou-se "Extremamente" ansiosa, com percentuais entre $57.1 \%$ no primeiro período e $67.4 \%$ no quarto. Chama a atenção o fato de, apesar da experiência na avaliação, os alunos do quarto período apresentarem os maiores níveis de ansiedade, indo contra mesmo a prerrogativa que ao realizar esse tipo de avaliação pela primeira vez o nível de ansiedade dos indivíduos pode ser maior quando comparado com indivíduos que já vivenciaram a mesma situação mais de uma vez (MOREIRA SNT, et al. 2015; FREDERICKS JA, et al. 2016; SILVA OS, et al. 2019). Ainda assim, não foram encontrados dados na literatura que comprovem que a ansiedade diminui após vivenciar mais de uma vez o OSCE, e se o nível de preparação antes da avaliação é maior no indivíduo com experiência pregressa.

Diversos fatores podem contribuir para tal condição (ansiedade), por exemplo, as novas responsabilidades exigidas, adaptação ao novo ambiente, frustração pela grande carga emocional aplicada, falta de preparo psicológico, privações das atividades de lazer (QUERIDO IA, 2014; RIBEIRO MGS, 2014; SANDERS J, et al. 2014; IFMSA, 2016; TENORIO LP, et al. 2016; CUNHA DHF., et al. 2017; MAYER FB, 2017).

Em um estudo, realizado por Wardley CS, et al. (2016), observou-se um nível de estresse muito alto durante a avaliação do método, sendo muitas vezes motivado pela distância da família, questões financeiras e o fator psicológíco a mediante a aproximação do ciclo clínico.

Já em relação ao grau de confiança, pôde-se observar que não houve diferença proporcional significativa entre os períodos. O quarto período mostrou maior proporção (51.2\%) em "Muito" e o segundo período em "Pouco" (64.6\%). Tais resultados podem ser reflexo de estratégias desenvolvidas pelo aluno, por exemplo, técnicas de estudos (método, tempo), preparação antecipada para a prova, boa relação professor-aluno, para que o mesmo possa se sentir confiante diante de uma avaliação e obter sucesso nesta (RIBEIRO MGS, 2014; MAYER FB, 2017). Na análise do desempenho no OSCE pelos discentes, foi observado que a maior parcela dos discentes entrevistados, de todos os períodos, atingiu o desempenho na avaliação como "Bom", em proporções acima de $50 \%$.

Os alunos do primeiro período alcançaram o melhor desempenho, com uma média de 8.33. O pior desempenho foi dos alunos do segundo período, com uma média de 7.36. Esse dado pode ser correlacionado tanto a escolha do método de estudo preferível por tais alunos, o qual baseia-se nos livros e anotações 
(79.2\%), o que pode não Ihes dar segurança no seguimento do exame físico, e também quanto ao grau de confiança atribuído, pois a maioria afirmou sentir-se pouco confiante antes da prova (64.6\%), podendo prejudicar o raciocínio clinico. Um estudo realizado numa instituição privada em Minas Gerais, encontrou dados que corroboram essa hipótese; nesse, a maioria dos discentes relatou a ansiedade como principal fator de interferência no desempenho acadêmico (ALVARENGA VC, et al., 2017).

Ainda referente ao desempenho da avaliação, o quarto período apresentou o segundo pior desempenho, com uma média de 7.63, mesmo em virtude da prática ter sido escolhida, pela grande maioria dos alunos (69.8\%), como a principal forma de estudo; do tempo dedicado aos estudos, pois destinam cerca de 3 ou $\geq 4$ horas; e devido a experiência adquirida ao longo dos semestres anteriores, tanto no direcionamento para a revisão da matéria, como nas habilidades clínicas. As razões aparentes para tal suposição baseiam-se no processo-aprendizagem do método PBL, já que não se caracteriza por ser um método acumulativo, ou seja, cada etapa, vivenciada pelo aluno, o prepara para aprender na próxima etapa (SILVA JOM, et al. 2018; COUTO LB, et al. 2019).

Ademais, o quarto período apresentou maior variação na nota atribuída ao desempenho no OSCE, o que pode ser consequência do nervosismo, tensão ou preocupação antes da prova (67.4\%), mediante ao extenso conteúdo didático, o qual deve ser discutido/praticado semanalmente com os professores e outros estudantes. Esse dado (67.4\%) é superior aos dados encontrados em uma tese de doutorado na Faculdade de Medicina da Universidade de São Paulo (USP) (MAYER FB, 2017), a qual mostrou que somente uma parte dos alunos (38\%), que responderam os questionários, sofre com preocupação excessiva.

A má qualidade do sono e a fatigabilidade também são fatores a serem abordados. Tanto esta como aquela podem ser reflexo do pouco horário disponível para estudo, fazendo com que isto ocorra quase exclusivamente no período noturno, prejudicando a qualidade do sono do estudante e ocasionando diversas repercussões à sua saúde, dentre elas a redução da qualidade de vida e a diminuição do desempenho acadêmico. Dados semelhantes foram verificados em uma pesquisa realizada na Universidade de São Paulo (USP), a qual mostrou que uma parcela considerável dos futuros médicos ${ }^{30}$, de todos os períodos do curso, sofre com má qualidade do sono (59.7\%) e cansaço (73,7\%).

Um dos pontos centrais que deve ser mencionado nesta pesquisa, são as possíveis fontes de viés que podem interferir na avaliação do aluno e, consequentemente, no seu desempenho. A avaliação do desempenho do aluno no OSCE deve ser independente do comportamento do examinador (brando ou severo), do gênero, raça e do interesse do aluno pelo assunto/domínio da estação, já que os fatores supracitados podem induzir uma avaliação injusta, não confiável ou incorreta sobre o nível de conhecimento e a habilidade prática do aluno (RIBEIRO MGS, 2014).

O OSCE, ao nível de avaliação, deve fornecer resultados válidos e confiáveis aos professores/examinadores, garantindo, desse modo, não somente a aprovação ou reprovação do aluno, mas também informações que permitam auxiliar o corpo docente a tomar decisões respaldadas se um aluno atingiu ou não um nível de proficiência clinica adequado para permitir uma prática segura fora do âmbito acadêmico (MAYER FB, 2017). Por fim, ressalta-se que esse estudo foi realizado em centro único e com número limitado de sujeitos, necessitando de pesquisas posteriores para corrigir ou corroborar os dados expostos.

\section{CONCLUSÃO}

O OSCE, assim como qualquer outro método avaliativo, apresenta determinados fatores que interferem no seu resultado final. Diante dessa avaliação, esse estudo demonstrou que, comparando-se os 4 semestres do ciclo básico: os métodos de estudos utilizados em maior proporção foram as anotações e os livros; a quantidade de tempo destinada aos estudos variou proporcionalmente entre 2 a 4 horas; a prevalência de ansiedade auto-referida "EXTREMAMENTE" foi comum entre os alunos do $1^{\circ}$ e $4^{\circ}$ semestre; o grau de confiança "MUITO" no conhecimento dos estudantes com o seu desempenho na avaliação foi prevalente entre os alunos do $4^{\circ}$ período, e o desempenho dos estudantes no OSCE foi melhor entre os alunos do $1^{\circ}$ semestre. 


\section{REFERÊNCIAS}

1. AlVARENGA VC, et al. Perfil dos estudantes dos dois primeiros anos do Curso de Medicina de uma instituição privada de ensino de Minas Gerais. Revista Saúde Dinâmica, 2019, 1(1):10-19.

2. BESTETTI RB, et al. Assessment Test Before the Reporting Phase of Tutorial Session in Problem-Based Learning. Advances in Medical Education and Practice. 2017.

3. COMERT M, et al.Assessing Communication Skills of Medical Students in Objective Structured Clinical Examinations (OSCE)-A Systematic Review of Rating Scales. PLOS ONE. 2016;(3):e0152717.

4. COUTO LB, et al. Formative assessment scores in tutorial sessions correlates with OSCE and progress testing scores in a PBL medical curriculum, Medical Education Online, 2019; 24:1, 1560862

5. CUNHA DHF, et. al. Percepção da qualidade de vida e fatores associados aos escores de qualidade de vida dos alunos de uma escola de medicina. J Bras Psiquiatr. 2017; 66(4): 189-96.

6. FILHO FABC, et al. Perfil do Estudante de Medicina da Universidade do Rio Grande do Norte (UERN). Rev. bras. educ. med., 2015, 39(1): 32-40.

7. FRANCOS CAGS, et al. OSCE para Competências de Comunicação Clínica e Profissionalismo: Relato de Experiência e Meta-Avaliação. Rev. bras. educ. med. 2015, 39(3):20-27.

8. FREDERICKSJA, et al. Student engagement, context, and adjustment: Addressing definitional, measurement, and methodological issues. Learning and Instruction. 2016; 43: 1-4.

9. GERMANO JCV. Perfil dos Estudantes do Curso de Medicina da Escola Multicampi de Ciências Médicas do Rio Grande do Norte/UFRN. Natal: Universidade Federal do Rio Grande do Norte. 2018.

10. GRAF J, et al. Communication skills of medical students during the OSCE: Gender-specific differences in a longitudinal trend study. BMC Medical Education. 2017; 17:75.

11. IFMSA. Saúde mental do estudante de medicina. International Federation of Medical Student's Associations of Brazil (IFMSA BRAZIL), novembro de 2016.

12. JOONG HS, et al. Students' performance in the different clinical skills assessed in OSCE: what does it reveal?, Medical Education Online, 2015; 20:1, 26185.

13. MAYER FB. A prevalência de sintomas de depressão e ansiedade entre os estudantes de medicina: um estudo multicêntrico no Brasil. 2017. Tese (Doutorado em Educação e Saúde). Faculdade de Medicina, Universidade de São Paulo, São Paulo, novembro de 2017

14. MESSIAS AJ, et al Caracterização dos Estudantes de Medicina da Universidade Federal da Fronteira Sul, Campus Chapecó. I Simpósio e II Semana Acadêmica UFFS Urgência e Emergência. 2018.

15. MOREIRA SNT, et al. Estresse na Formação médica: Como Lidar com Essa realidade? Rev. bras. educ. med. 2015, 39(4): 558-564.

16. MINELLA LS. Medicina e feminização em universidades brasileiras: o gênero nas interseções. Rev. Estud. Fem., 2017, 25(3): 1111-1128.

17. NETO JAC, et al. Fontes de Estudo e Pesquisa Entre os Estudantes de Medicina. Rev. Med. Minas Gerais 2016; 26:e1787.

18. NEVES RS, et al. Avaliação do Exame Clínico Objetivo Estruturado (OSCE) por Estudantes e Docentes de Graduação em Enfermagem. Com. Ciências Saúde. 2016; 27(4): 309-316.

19. PAES AT, et al. Perfil dos ingressantes na primeira turma de graduação em Medicina da Faculdade Israelita de Ciências da Saúde Albert Einstein. Einstein (São Paulo). 2018;16(3):eAO4228.

20. RADHIKA G, et al. Perceptions of the introduction of objective structured practical examination (OSPE)/objective structured clinical examination (OSCE): A pilot study carried out in Government Medical College, Ananthapuramu, Andhra Pradesh, India. J NTR Univ Health Sci 2015; 4:145-9.

21. RIBEIRO MGS. Sofrimento psíquico entre estudantes de medicina da UFMG: uma contribuiçao da Assessoria de Escuta Acadêmica [dissertaçao]. Belo Horizonte: Universidade Federal de Minas Gerais, Faculdade de Medicina; 2014.

22. SANDERS J, et al. Developmental student support in undergraduation medical education: AMEE Guide no 92 . Med Teach, 2014, 36(12): 1016-1026.

23. SCHEFFER M, et al. Demografia Médica no Brasil 2018. São Paulo, SP: FMUSP, CFM, Cremesp, $2018: 286$.

24. SILVAOS, et al. A influência da ansiedade no desempenho acadêmico no exame clínico objetivo estruturado (OSCE). Braz Oral Res. 2019, 33(2): 20-25.

25. SILVA JOM, et al. Engajamento Entre Estudantes do Ensino Superior nas Ciências da Saúde (Validação do Questionário Ultrecht Work Engagement Scale (UWES-S) Com Estudantes do Ensino Superior nas Ciências da Saúde). Ver. Brasileira de Educação Médica. 2018; 42 (2): 15-25.

26. QUERIDO IA. Estresse, personalidade e habilidades sociais de estudantes de medicina no internato. $2014.98 \mathrm{f}$. Dissertação (Mestrado Profissional em Ensino na Saúde) - Universidade Federal de Goiás, Goiânia, 2014

27. TENORIO LP, et al. Saúde Mental de Estudantes de Escolas Médicas com Diferentes Modelos de Ensino. Rev. bras. educ. med. Rio de Janeiro, 2016, 40 (4): 574-582.

28. WARDLEY CS, et al. Comparison of Students' Perceptions of Stress in Parallel Problem-Based and Lecture-Based Curricula. J Physician Assist Educ. 2016 Mar;27(1):7-16.

29. ZIMMERMANN MH. Avaliação Clínica Objetiva Estruturada (OSCE) com Feedback Efetivo e Vídeo Efetivo: Sua Interface no Ensino e na Aprendizagem. Ponta Grossa: Universidade Tecnológica Federal do Paraná. 2019 